Pacific Journal of Mathematic 


\title{
ZONAL MULTIPLIERS ON THE HEISENBERG GROUP
}

\author{
GiAnCARLO MAUCERI
}

\begin{abstract}
We investigate $L^{p}$ boundedness of zonal multipliers on the $2 n+1$-dimensional Heisenberg group $H^{n}$. These are multipliers which are invariant under the group $S U(n)$ acting in the noncentral variables. The result is then applied to derive sufficient conditions for $L^{p}$ boundedness for a class of multipliers associated with $S U(n)$ invariant operators in the enveloping algebra of $H^{n}$. A necessary condition is also obtained with the aid of group contractions.
\end{abstract}

1. Introduction. The purpose of this paper is the extension to higher dimensional Heisenberg groups of some of the results of [4]. The main tools in the proof of the multiplier theorem in [4] are the machinery developed by Coifman and Weiss in [1] and the Fourier analysis on the Heisenberg group [9].

Both of them are available in the more general case of the $2 n+$ 1-dimensional Heisenberg group $\boldsymbol{H}^{n}$. However it turns out that a strict imitation of the methods of [4], if at all possible, would produce prohibitively long and involved proofs.

These difficulties can be avoided if we restrict our attention to the sub algebra of multipliers which are invariant under the action of the group $S U(n)$ acting in the noncentral variables.

We call these multipliers zonal multipliers. It turns out that zonal multipliers are convolution operators with a zonal tempered distribution on $\boldsymbol{H}^{n}$.

One can show that these convolution operators on $\boldsymbol{H}^{n}$ may be realized as averages over $S U(n)$ of convolution operators transferred from $\boldsymbol{H}^{n-1}$. Thus we reduce the problem of finding sufficient conditions for their boundedness in $L^{p}\left(\boldsymbol{H}^{n}\right)$ to the corresponding problem in $\boldsymbol{H}^{n-1}$. A repeated application of this argument will enable us eventually to apply the multiplier theorem in [4].

This method, which is a generalization of the classical method of rotation used by Calderòn and Zygmund to deal with singular integrals with odd kernels, is due to Coifman and Weiss, who applied it to several problems in harmonic analysis [2].

Next, in $\S 4$, we consider a particular algebra of zonal multipliers on $H^{n}$ defined in terms of their symbol $F$, which is a function defined in the sectors $S_{n}=\left\{(\lambda, r) \in \boldsymbol{R}^{2}: r \geqq n|\lambda|>0\right\}$. For these operators the conditions of the multiplier theorem assume a particularly simple form, being expressed in terms of growth conditions on the derivatives of the symbol. 
In the last section we investigate a relationship between $L^{p}$ multipliers of $\boldsymbol{H}^{n}$ and $L^{p}$ multipliers of $\boldsymbol{C}^{n}$ with the aid of group contractions.

Some of the results of this paper were announced without proofs in [3].

2. Fourier analysis on the Heisenberg group. In this section we recall the basic facts of harmonic analysis on the Heisenberg group. The main references here are the papers [8] and [9] of Geller.

The $2 n+1$-dimensional Heisenberg group $\boldsymbol{H}^{n}$ is the nilpotent Lie group whose underlying manifold is $\boldsymbol{R} \times \boldsymbol{C}^{n}$. The multiplication is given by:

$$
(t, z)\left(t^{\prime}, z^{\prime}\right)=\left(t+t^{\prime}+\Phi\left(z, z^{\prime}\right), z+z^{\prime}\right), \quad t \in \boldsymbol{R}, z, z^{\prime} \in \boldsymbol{C}^{n}
$$

where $\Phi$ is the skew-symmetric bilinear form $\Phi\left(z, z^{\prime}\right)=2 \mathscr{T} m \sum{ }_{j} \bar{z}_{j}^{\prime}$ on $\boldsymbol{C}^{n} \times \boldsymbol{C}^{n}$. Sometimes we shall also use coordinates $(t, x, y) \in \boldsymbol{R} \times$ $\boldsymbol{R}^{2 n}$, where $z=x+i y$. The Haar measure is the Lebesgue measure $d t d z d \bar{z}$ on $\boldsymbol{R} \times \boldsymbol{C}^{n}$.

The group $S U(n)$ acts as group of automorphisms of $\boldsymbol{H}^{n}$ in the following way:

$$
u \cdot(t, z)=(t, u \cdot z)
$$

for $u \in S U(n),(t, z) \in \boldsymbol{H}^{n}$.

We say that a function $f: \boldsymbol{H}^{n} \rightarrow \boldsymbol{C}$ is zonal if $f$ is invariant under the action of $S U(n)$.

It is well known that the infinite dimensional representations of $\boldsymbol{H}^{n}$ are parametrized by $\boldsymbol{R}^{*}$, the set of nonzero real numbers, and that they can be realized on $L^{2}\left(\boldsymbol{R}^{n}\right)$ as follows. For every $\lambda \in \boldsymbol{R}^{*}$, let $\pi_{\lambda}$ be the corresponding representation. Then for $\varphi \in L^{2}\left(\boldsymbol{R}^{n}\right)$ :

$$
\pi_{\lambda}(t, x, y) \varphi(\xi)=e^{i \lambda t} e^{2 i \lambda\langle 2 \xi-x, y\rangle} \varphi(\xi-x) .
$$

Given a function $f \in L^{1}\left(\boldsymbol{H}^{n}\right)$ the Fourier transform of $f$ is the operator valued function:

$$
\hat{f}(\lambda)=\int_{\boldsymbol{H}^{n}} f(t, z) \pi_{\lambda}(t, z) d t d z d \bar{z} .
$$

Choosing an appropriate orthonormal basis in the space of each representation $\pi_{\lambda}$, one can give an explicit expression of the matrix coefficients of operators $\hat{f}(\lambda)$ in terms of $f$.

For every $\lambda \in R^{*}$ let $\left\{\mathscr{C}_{\alpha, \lambda}: \alpha \in N^{n}\right\}$ be the orthonormal basis in $L^{2}\left(\boldsymbol{R}^{n}\right)$ defined by 


$$
\mathscr{H}_{\alpha, \lambda}(\xi)=\prod_{j=1}^{n} \mathscr{H}_{\alpha_{j}, \lambda}\left(\xi_{j}\right), \alpha=\left(\alpha_{1}, \cdots, \alpha_{n}\right) \in N^{n}, \xi=\left(\xi_{1}, \cdots, \xi_{n}\right) \in \boldsymbol{R}^{n} .
$$

Here

$$
\mathscr{H}_{k, \lambda}(s)=\left(2|\lambda|^{1 / 2}\right)^{1 / 2} \mathscr{H}_{k}\left(2|\lambda|^{1 / 2} s\right)
$$

is the Hermite function $\mathscr{H}_{k}$ scaled by a factor $2|\lambda|^{1 / 2}$.

Given multiindices $m \in Z^{n}, \alpha \in N^{n}$, we shall use the notation:

$$
\begin{array}{ll}
m_{i}^{+}=\max \left(m_{i}, 0\right) ; & m_{i}^{-}=-\min \left(m_{i}, 0\right) ; \\
m^{+}=\left(m_{1}^{+}, \cdots, m_{n}^{+}\right) ; & m^{-}=\left(m_{1}^{-}, \cdots, m_{n}^{-}\right) \\
|m|=\sum m_{i} ; & \alpha !=\alpha_{1} ! \cdots \alpha_{n} ! \\
\alpha \pm m=\left(\alpha_{1} \pm m_{1}, \cdots,\right. & \left.\alpha_{n} \pm m_{n}\right) .
\end{array}
$$

Define the partial isometries $W_{\alpha}^{m}(\lambda)$ on $L^{2}\left(\boldsymbol{R}^{n}\right)$ by:

$$
\begin{aligned}
& W_{\alpha}^{m}(\lambda) \mathscr{H}_{\beta, \lambda}=(-1)^{|m|+} \delta_{\alpha+m^{+}, \beta} \mathscr{H}_{\alpha^{+m^{-}, \lambda}} \text { for } \lambda>0 \\
& W_{\alpha}^{m}(\lambda)=W_{\alpha}^{m}(-\lambda)^{*} \text { for } \lambda<0 .
\end{aligned}
$$

Then $\left\{W_{\alpha}^{m}(\lambda): m \in \boldsymbol{Z}^{n}, \alpha \in N^{n}\right\}$ is for every $\lambda \in \boldsymbol{R}^{*}$ an orthonormal basis in the Hilbert space of Hilbert-Schmidt operators on $L^{2}\left(\boldsymbol{R}^{n}\right)$.

Given a function $f$, to find $\hat{f}$ write:

$$
f(t, z)=\sum_{m \in \mathbf{Z}^{n}} f_{m}\left(t, r_{1}, \cdots, r_{n}\right) \exp \left(i \sum m_{k} \theta_{k}\right)
$$

where $z_{j}=r_{j} e^{i \theta_{j}}$, and denote by $\mathscr{F}_{1} f_{m}$ the Euclidean Fourier transform of $f_{m}$ with respect to the variable $t$, i.e.:

$$
\mathscr{F}_{1} f_{m}\left(\lambda, r_{1}, \cdots, r_{n}\right)=\int_{R} e^{i \lambda t} f_{m}\left(t, r_{1}, \cdots, r_{n}\right) d t
$$

Then, denoting by $L_{j}^{k}$ the Laguerre polynomial of degree $j$ and type $k$ and by

$$
l_{j}^{k}(s)=\left(\frac{j !}{(j+k) !}\right)^{1 / 2} s^{k / 2} L_{j}^{k}(s) e^{-s / 2}
$$

the corresponding Laguerre function, we have:

$$
\hat{f}(\lambda)=\sum_{m \in Z^{n}} \sum_{\alpha \in N^{n}} R_{\alpha}^{m}(\lambda ; f) W_{\alpha}^{m}(\lambda)
$$

where

$$
R_{\alpha}^{m}(\lambda ; f)=(2 \pi)^{n} \int_{R_{+}^{n}} \mathscr{F}_{1} f_{m}\left(\lambda, r_{1}, \cdots, r_{n}\right) \prod_{k=1}^{n} l_{\alpha_{k}}^{\left\langle m_{k} !\right.}\left(2|\lambda| r_{k}^{2}\right) r_{k} d r_{k} .
$$

The inversion formula is: 


$$
\begin{aligned}
& f\left(t, z_{1}, \cdots, z_{n}\right) \\
& \quad=\frac{2^{n-1}}{\pi^{n+1}}\left[\int_{R} e^{-i \lambda t} \sum_{m, \alpha} R_{\alpha}^{m}(\lambda ; f) \prod_{k=1}^{n} l_{\alpha_{k}}^{m_{k}}\left(2|\lambda| r_{k}^{2}\right)|\lambda|^{n} d \lambda \exp \left(i \sum m_{k} \theta_{k}\right)\right] .
\end{aligned}
$$

For a zonal function $f(t, z)=f_{0}(t,|z|)$ one has $R_{\alpha}^{m}(\lambda ; f)=0$ for $m \neq 0$ and $R_{\alpha}^{\prime}(\lambda ; f)=R_{\beta}^{\prime}(\lambda ; f)$ if $|\alpha|=|\beta|[8]$. Hence we write $R_{N}(\lambda ; f)$ for $R_{\alpha}^{0}(\lambda ; f)$ when $f$ is zonal and $|\alpha|=N$.

Now if we denote by $P_{N}^{(n)}(\lambda)$ the projection on the $\left(\begin{array}{c}N+n-1 \\ n-1\end{array}\right)$ dimensional subspace of $L^{2}\left(\boldsymbol{R}^{n}\right)$ spanned by $\left\{\mathscr{H}_{\alpha, \lambda}:|\alpha|=N\right\}, N \in N$, it is not difficult to see that

$$
\hat{f}(\lambda)=\sum_{N \in N} R_{N}(\lambda ; f) P_{N}^{(n)}(\lambda)
$$

where

$$
\begin{aligned}
& R_{N}(\lambda ; f) \\
& \quad=\omega_{2 n-1} \frac{N !}{(N+n-1) !} \int_{0}^{+\infty} \mathscr{F}_{1} f_{0}(\lambda, r) L_{N}^{n-1}\left(2|\lambda| r^{2}\right) e^{-|\lambda| r^{2}} r^{2 n-1} d r
\end{aligned}
$$

and $\omega_{2 n-1}$ is the surface area of the unit sphere in $\boldsymbol{R}^{2 n}$. The inversion formula becomes:

$$
f_{0}(t, r)=\frac{2^{n-1}}{\pi^{n+1}} \int_{R} e^{-i \lambda t} \sum_{N \in N} R_{N}(\lambda ; f) L_{N}^{n-1}\left(2|\lambda| r^{2}\right) e^{-|\lambda| r^{2}}|\lambda|^{n} d \lambda .
$$

We also have the following Plancherel formula:

$$
\|f\|_{2}^{2}=\frac{2^{n-1}}{\pi^{n+1}} \int_{R} \sum_{N \in N}\left|R_{N}(\lambda: f)\right|^{2} \frac{(N+n-1) !}{N !}|\lambda|^{n} d \lambda .
$$

3. Zonal multipliers. A left multiplier of $L^{p}\left(\boldsymbol{H}^{n}\right), 1 \leqq p \leqq \infty$, is an operator valued function $M$ defined on $\boldsymbol{R}^{*}$ and taking values in $\mathscr{B}\left(L^{2}\left(\boldsymbol{R}^{n}\right)\right)$, the algebra of bounded operators on $L^{2}\left(\boldsymbol{R}^{n}\right)$, such that the mapping $f \mapsto M f$ defined by

$$
(M f)^{\wedge}(\lambda)=M(\lambda) \hat{f}(\lambda) \quad \lambda \in \boldsymbol{R}^{*}, f \in L^{1} \cap L^{p}\left(\boldsymbol{H}^{n}\right)
$$

extends to a bounded linear operator on $L^{p}\left(\boldsymbol{H}^{n}\right)$. Right multipliers are also defined in an obvious way.

We say that a multiplier $M$ is zonal if $M$ commutes with the action of $S U(n)$ on $L^{p}\left(\boldsymbol{H}^{n}\right)$.

It is not difficult to prove, using the fact that every $L^{p}$ multiplier is an $L^{2}$ multiplier [10] and the Plancherel formula, that $M$ is zonal if and only if

$$
M(\lambda)=\sum_{N \in \boldsymbol{N}} R_{N}(\lambda) P_{N}^{(n)}(\lambda) \quad \lambda \in \boldsymbol{R}^{*},
$$

where $\left\{R_{N}: N \in N\right\}$ is a uniformly bounded sequence of measurable 
functions of $\lambda \in \boldsymbol{R}^{*}$. It is not hard to see also that if $M$ is zonal then $M$ is a left $L^{p}$ multiplier if and only if $M$ is a right $L^{p}$ multiplier. Therefore in the following we will not distinguish any more between left and right zonal multipliers.

It is well known that $L^{p}$ multipliers are operators of convolution by tempered distributions on $\boldsymbol{H}^{n}$ [12]. The following theorem shows that, at least when such a distribution is a continuous zonal function with compact support, the question of the $L^{p}$ boundedness of the corresponding operator can be reduced to the analogous question in lower dimension.

THeOREM 3.1. Suppose $k_{0}$ is a continuous function on $\boldsymbol{R} \times \boldsymbol{R}^{+}$ having compact support. It the kernel $k(t, z)=|z|^{2} k_{0}(t,|z|)$ defines a bounded convolution operator on $L^{p}\left(\boldsymbol{H}^{n-1}\right)$ with norm $N_{p}$, then the kernel $h(t, z)=k_{0}(t,|z|)$ defines a bounded convolution operator on $L^{p}\left(\boldsymbol{H}^{n}\right)$ with norm not exceeding $\left(\omega_{2 n-1} / \omega_{2 n-3}\right) N_{p}$, where $\omega_{k-1}$ is the surface area of the unit sphere in $\boldsymbol{R}^{k}$.

Proof. The proof is based on an extension of the rotation method of Calderon-Zygmund due to Coifman and Weiss [2]. We give a sketch of the argument for completeness.

Given a function $f \in L^{p}\left(\boldsymbol{H}^{n}\right)$, first one shows that it is possible to express the convolution $h * f$ as an average over $S U(n)$ of certain transferred operators on $L^{p}\left(\boldsymbol{H}^{n}\right)$. Then one shows that the $L^{p}$ norm of the transferred operators can be bounded by $N_{p}$. The conclusion then follows by an application of Minkowski's integral inequality. More precisely one has:

$$
h * f\left(t_{1}, z_{1}\right)=\frac{\omega_{2 n-1}}{\omega_{2 n-3}} \int_{S U(n)} d u \int_{\left[I^{n-1}\right.} k(t, z) U_{(t, z)}^{u} f\left(t_{1}, z_{1}\right) d t d z d \bar{z}
$$

where for every fixed $u \in S U(n) U^{u}$ is the isometric representation of $\boldsymbol{H}^{n-1}$ on $L^{p}\left(\boldsymbol{H}^{n}\right)$ defined by:

$$
U_{(t, z)}^{u} f\left(t_{1}, z_{1}\right)=f\left((t, u z)^{-1}\left(t_{1}, z_{1}\right)\right) .
$$

Note that we are identifying $\boldsymbol{H}^{n-1}$ with the subgroup of the elements of $\boldsymbol{H}^{n}$ of the form $\left(t, z_{1}, \cdots, z_{n-1}, 0\right)$.

Since $S U(n)$ acts as a group of automorphisms of $\boldsymbol{H}^{n}$ it is easily checked that $U^{u}$ is a representation of $\boldsymbol{H}^{n-1}$.

The fact that the norm of the transferred operator

$$
U^{u}(k) f=\int_{H^{n-1}} k(t, z) U_{(t, z)}^{u} f d t d z d \bar{z}
$$

is bounded by the norm of the operator of convolution by $k$ on 
$L^{p}\left(\boldsymbol{H}^{n-1}\right)$ then follows from the general result on transference [2] and the amenability of $\boldsymbol{H}^{n-1}$.

Now we want to use Theorem 3.1 to deduce sufficient conditions for the boundedness of a generalized convolution operator in terms of the corresponding multiplier.

In this connection we introduce the difference operator $\mathscr{D}_{n}$ defined on sequences $\left\{R_{N}: N \in N\right\}$ of functions of $\lambda \in R^{*}$ by:

$$
\begin{aligned}
& \mathscr{D}_{n} R_{N}(\lambda)=(N+n-1) R_{N}(\lambda)+N R_{N-1}(\lambda) \text { for } N \neq 0 \\
& =(n-1) R_{0}(\lambda) \quad \text { for } N=0 \text {. }
\end{aligned}
$$

THEOREM 3.2. Let $M(\lambda)=\sum_{N \in N} R_{N}(\lambda) P_{N}^{(n)}(\lambda), n \geqq 2$, where $\left\{R_{N}: N \in N\right\}$ is a uniformly bounded sequence of complex valued continuous functions of $\lambda \in \boldsymbol{R}^{*}$, and

$$
m(\lambda)=\sum_{N \in N} \mathscr{D}_{n} R_{N}(\lambda) P_{N}^{(n-1)}(\lambda)
$$

If the operator valued function $m$ defines a zonal multiplier of $L^{p}\left(\boldsymbol{H}^{n-1}\right)$ then $M$ defines a zonal multiplier of $L^{p}\left(\boldsymbol{H}^{n}\right)$.

Proof. Assume first that $M$ is the Fourier transform of a continuous function $h(t, z)=k_{0}(t,|z|)$ with compact support. Then:

$$
R_{N}(\lambda)=\omega_{2 n-1} \frac{N !}{(N+n-1) !} \int_{0}^{+\infty} \mathscr{F}_{1} k_{0}(t, r) L_{N}^{n-1}\left(2|\lambda| r^{2}\right) e^{-|\lambda| r^{2}} r^{2 n} d r .
$$

If the kernel $k(t,|z|)=|z|^{2} \boldsymbol{k}_{0}(t,|z|)$ defines a bounded convolution operator on $L^{p}\left(\boldsymbol{H}^{n-1}\right)$, then by Theorem 3.1, $h$ defines a bounded convolution operator on $L^{p}\left(\boldsymbol{H}^{n}\right)$. The Fourier transform of $k$ is

$$
\hat{k}(\lambda)=\sum_{N \in N} R_{N}(\lambda ; k) P_{N}^{(n-1)}(\lambda)
$$

where

$$
\begin{aligned}
R_{N}(\lambda ; k) & \\
= & \omega_{2 n-3} \frac{N !}{(N+n-2) !} \int_{0}^{+\infty} \mathscr{F}_{1} k_{0}(t, r) r^{2} L_{N}^{n-2}\left(2|\lambda| r^{2}\right) \\
& \quad \exp \left(-|\lambda| r^{2}\right) r^{2 n-2} d r .
\end{aligned}
$$

Therefore from the classical formulas (see [5]):

$$
\begin{aligned}
& L_{N}^{n-2}(x)=L_{N}^{n-1}(x)-L_{N-1}^{n-1}(x) \quad N \geqq 1 \\
& L_{0}^{n-2}(x)=L_{0}^{n-1}(x)=1
\end{aligned}
$$

it is easily seen that 
and

$$
R_{N}(\lambda ; k)=\frac{\omega_{2 n-3}}{\omega_{2 n-1}}\left[(N+n-1) R_{N}(\lambda)-N R_{N-1}(\lambda)\right] \text { for } \quad N \geqq 1
$$

$$
R_{0}(\lambda ; k)=\frac{\omega_{2 n-3}}{\omega_{2 n-1}}(n-1) R_{0}(\lambda) .
$$

This proves the theorem when $M$ is the Fourier transform of a continuous function with compact support. For the general case we shall show that $M$ can be appropriately approximated by multipliers which are Fourier transforms of continuous functions with compact support.

Given a function $f$ defined on $\boldsymbol{H}^{n}$ let

$$
\begin{aligned}
& \mathscr{F}_{1,2} f(\tau, \xi, \eta) \\
& \quad=\int_{a^{\prime n}} \exp [i(t \tau+\langle\xi, x\rangle+\langle\eta, y\rangle)] f(t, x, y) d t d x d y
\end{aligned}
$$

be its Euclidean Fourier transform. The following lemma gives the connections between the different transforms of a zonal function.

Lemma 3.3. Suppose $f$ is a zonal function in $L^{2}\left(\boldsymbol{H}^{n}\right)$; thus $f(t, z)=f_{0}(\dot{t},|z|)$ and $\mathscr{F}_{1,2} f(\tau, \zeta)=\left(\mathscr{F}_{1,2} f\right)_{0}(\tau,|\zeta|)$.

Then for almost every $\tau, \rho, \lambda$

$$
\begin{aligned}
&\left(\mathscr{F}_{1,2} f\right)_{0}(\tau, \rho)=C_{n} \sum_{N \in N} R_{N}(\lambda ; f) L_{N}^{n-1}\left(\rho^{2} / 2|\tau|\right) \exp \left(-\rho^{2} / 4|\tau|\right) \\
& R_{N}(\lambda ; f)= \gamma_{n}(2|\lambda|)^{-n} \frac{N !}{(N+n-1) !} \int_{0}^{+\infty}\left(\mathscr{F}_{1,2} f\right)_{0}(\lambda, s) L_{N}^{n-1}\left(s^{2} / 2|\lambda|\right) \\
& \times \exp \left(-s^{2} / 4|\lambda|\right) s^{2 n-1} d s
\end{aligned}
$$

where both the series and the integral converge in $L^{2}$.

Proof. Assume first that $f$ is in $\mathscr{S}\left(\boldsymbol{H}^{n}\right)$, the Schwartz space of fast decreasing functions on $H^{n}$. Since it is easily seen that the Fourier transform in $\boldsymbol{R}^{2 n}$ of $L_{N}^{n-1}\left(2|\lambda||z|^{2}\right) \exp \left(-|\lambda||z|^{2}\right)$ is

$$
(2|\lambda|)^{-n} L_{N}^{n-1}\left(|\zeta|^{2} / 2|\lambda|\right) \exp \left(-|\zeta|^{2} / 4|\lambda|\right),
$$

taking the Fourier transform $\mathscr{F}_{1,2}$ of both sides of the Fourier inversion formula (2.5) we get (3.1). A similar argument yields (3.2). The general case $f \in L^{2}\left(\boldsymbol{H}^{n}\right)$ follows by a limit argument.

Lemma 3.4. Suppose $M$ satisfies the hypothesis of Theorem 3.2. Then there exists a family $\left\{k_{\varepsilon, \sigma}: \varepsilon, \sigma>0\right\}$ of continuous zonal functions with compact support such that:

(i) $M(\lambda)$ is in the weak operator closure of $\left\{\hat{k}_{\varepsilon, \sigma}(\lambda): \varepsilon, \sigma>0\right\}$ for every $\lambda \in \boldsymbol{R}^{*}$, 
(ii) The $L^{p}$ multiplier norms of $\hat{k}_{\varepsilon, \sigma}$ are bounded by a constant times the $L^{p}$ multiplier norm of $M$.

Proof. First we show that $M$ is the strong operator limit of operators $T_{\varepsilon}, \varepsilon>0$, where $T_{\varepsilon} f=k_{\varepsilon} * f$ is the operator of convolution by a continuous functions $k_{\varepsilon}$ in $L^{2}\left(\boldsymbol{H}^{n}\right)$. Moreover $k_{\varepsilon}$ can be chosen so that the norm of $T_{\varepsilon}$ on $L^{p}\left(\boldsymbol{H}^{n}\right)$ is bounded by a constant times the norm of $M$ on $L^{p}\left(\boldsymbol{H}^{n}\right)$ and for every $\lambda \in \boldsymbol{R}^{*} M(\lambda)$ is in the weak operator closure of $\left\{\hat{k}_{\varepsilon}(\lambda): \varepsilon>0\right\}$.

Let $\varphi_{\varepsilon}$ be the function defined by:

$$
\hat{\varphi}_{\varepsilon}(\lambda)=\sum_{N \in N} e^{-\varepsilon(2 N+1)^{2}|\lambda|^{2}} P_{N}^{(n)}(\lambda) .
$$

By [9, Theorem 1] $\rho_{\varepsilon} \in \mathscr{S}\left(\boldsymbol{H}^{n}\right)$ for every $\varepsilon>0$.

Moreover from (2.4) it follows easily that

$$
\varphi_{\varepsilon}(t, z)=\varepsilon^{-(n+1) / 2} \varphi_{1}\left(\varepsilon^{-1 / 2} t, \varepsilon^{-1 / 4} z\right) .
$$

By (2.4) and the Lebesgue dominated convergence theorem $\int_{H^{n}} \varphi_{\varepsilon} d t d z d \bar{z}=$ $\lim _{\lambda \rightarrow 0} R_{N}\left(\lambda ; \varphi_{\varepsilon}\right)=1$. Hence $\left\{\varphi_{\varepsilon}: \varepsilon>0\right\}$ is an approximate identity for the convolution.

Let $\mathrm{Conv}_{p}, 1 \leqq p \leqq \infty$, denote the Banach algebra of all bounded operators on $L^{p}\left(\boldsymbol{H}^{n}\right)$ commuting with right translations. It is well known that $\operatorname{Conv}_{p}$ can be identified with the algebra of left multipliers of $L^{p}\left(\boldsymbol{H}^{n}\right)$ and that $\operatorname{Conv}_{p} \subset \mathrm{Conv}_{2}$ for every $1 \leqq p \leqq+\infty$ [10]. Hence the function $k_{\varepsilon}=M \varphi_{\varepsilon}$ is in $L^{2}\left(\boldsymbol{H}^{n}\right)$. Moreover, since $k_{\varepsilon}=$ $M\left(\varphi_{\varepsilon / 2} * \varphi_{\varepsilon / 2}\right)=\left(M \varphi_{\varepsilon / 2}\right) * \varphi_{\varepsilon / 2}, k_{\varepsilon}$ is also a continuous function. It is also obvious that $M(\lambda)$ is in the weak operator closure of $\left\{\hat{k}_{\varepsilon}(\lambda): \varepsilon>0\right\}$. Let $T_{\varepsilon}$ be the operator of left convolution by $k_{\varepsilon}$. Then $\|T\|_{\mathrm{Conv}_{p}} \leqq$ $\|M\|_{\text {Conv } p}\left\|\varphi_{1}\right\|_{1}$.

To finish the proof of the lemma we need to recall some facts about the algebras $A_{p}$. For $p=1, A_{1}$ is the space $C_{0}\left(\boldsymbol{H}^{n}\right)$ of the continuous functions vanishing at infinity. For $p>1, A_{p}$ is the smallest Banach space of continuous functions on $\boldsymbol{H}^{n}$ containing all the functions of the form $f=u * v, u \in L^{p^{\prime}}\left(\boldsymbol{H}^{n}\right), v \in L^{p}\left(\boldsymbol{H}^{n}\right), p, p^{\prime}$ conjugate exponents. It is well known [11] that $A_{p}$ is a Banach algebra for the pointwise multiplication and that $\operatorname{Conv}_{p}$ is the Banach dual of $A_{p}$, via the pairing:

$$
\langle T, u * v\rangle=\langle u, T \ddot{v}\rangle .
$$

The algebra $A_{p}$ operates on $\mathrm{Conv}_{p}$ as follows:

$$
\langle\varphi \cdot T, f\rangle=\langle T, \varphi \cdot f\rangle
$$

for $T \in \operatorname{Conv}_{p}, \varphi, f \in A_{p}$. In particular when $T$ is the convolution by 
a function $h \in L^{1}\left(\boldsymbol{H}^{n}\right)$, the product $\phi \cdot T$ corresponds to the ordinary product $\varphi h$. Now let $\varphi$ be a zonal $C^{\infty}$ function on $\boldsymbol{H}^{n}$, with compact support, such that $\varphi \geqq 0$ and $\|\varphi\|_{2}=1$. Define $F_{1}=\varphi * \varphi$, and $F_{\sigma}(t, z)=F_{1}\left(\sigma^{2} t, \sigma z\right)$, for $\sigma>0$. Then $F_{\sigma}$ is in $A_{p}$ and its norm is bounded by a constant $c_{p}$ independent of $\sigma$.

Let $k_{\varepsilon, \sigma}=F_{\sigma} k_{\varepsilon}$. We claim that the family $\left\{k_{\varepsilon, \sigma}: \varepsilon, \sigma>0\right\}$ satisfies the conclusion of the lemma. Clearly $k_{\varepsilon, \sigma}$ is continuous and has compact support. Moreover since for every $f \in A_{p}$ :

$$
\left|\left\langle F_{o} \cdot T_{\varepsilon}, f\right\rangle\right|=\left|\left\langle T_{\varepsilon}, F_{\sigma} f\right\rangle\right| \leqq C_{p}\|M\|_{\mathrm{Conv}_{p}}\|f\|_{A_{p}}
$$

the norm of $\left(F_{o} k_{\varepsilon}\right)^{\wedge}$ as $L^{p}$ multiplier does not exceed $C_{p}\|M\|_{\mathrm{Conv}_{p}}$. To prove (i) observe first that the Euclidean Fourier transform of $k$ is a continuous function vanishing at infinity in the strip $\{(\lambda, \zeta)$ : $\left.0<r_{0} \leqq|\lambda|<r_{1}\right\}$. Indeed by Lemma $3.3\left(\mathscr{F}_{1,2} k_{\varepsilon}\right)(\lambda, \zeta)=\left(\mathscr{F}_{1,2} k_{\varepsilon}\right)_{0}(\lambda,|\zeta|)$ where

$$
\begin{aligned}
& \left(\mathscr{F}_{1,2} k_{\varepsilon}\right)_{0}(\lambda, \rho) \\
& \quad=c_{n} \sum_{N \in N} R_{N}(\lambda) \exp \left[-\varepsilon(2 N+1)^{2}|\lambda|^{2}\right] L_{N}^{n-1}\left(\rho^{2} / 2|\lambda|\right) \exp \left(-\rho^{2} / 4|\lambda|\right) .
\end{aligned}
$$

From the inequality (see [5]):

$$
\left|L_{N}^{k}(x)\right| e^{-x / 2} \leqq \frac{(N+k) !}{N ! k !} \leqq(1+k)^{N} \quad \text { for } \quad k \geqq 0
$$

and the assumptions on $R_{N}(\lambda)$ it follows that the series is uniformly convergent in the strip $\left\{(\lambda, \zeta): r_{0} \leqq|\lambda| \leqq r_{1}\right\}$ to a continuous function vanishing at infinity. On the other hand since $F_{\sigma}(0,0)=\|\varphi\|_{2}=1$ and $\left\|\mathscr{F}_{1,2} F\right\|_{1}$ is bounded by a constant independent of $\sigma,\left\{\mathscr{F}_{1,2} F_{\sigma}: \sigma>0\right\}$ is an approximate identity for the ordinary convolution in $\boldsymbol{R}^{2 n+1}$. Thus $\mathscr{F}_{1,2}\left(F_{\sigma} k_{\varepsilon}\right)=\mathscr{F}_{1,2} F_{\sigma} * \mathscr{F}_{1,2} k$ converges uniformly to $\mathscr{F}_{1,2} k_{\varepsilon}$ in the strip $\left\{(\lambda, \rho): r_{0} \leqq|\lambda| \leqq r_{1}\right\}$. Hence by (3.2) and the Lebesgue dominated convergence theorem $R_{N}\left(\lambda ; k_{\varepsilon}\right)=\lim _{\sigma \rightarrow \infty} R_{N}\left(\lambda, F_{o} k_{\varepsilon}\right)$. So $M(\lambda)$ is in the weak operator closure of the set $\left\{\hat{k}_{\varepsilon, \sigma}(\lambda): \varepsilon, \sigma>0\right\}$ and the lemma is proved.

The proof of Theorem 3.2 now follows by an approximation argument. We omit the details.

REMARK. The same arguments in the proofs of Theorems 3.1 and 3.2 can be used to obtain weak type $(p-p)$ estimates for the multiplier $M$ on $L^{p}\left(\boldsymbol{H}^{n}\right)$ from the corresponding estimates for $m$ on $L^{p}\left(\boldsymbol{H}^{n-1}\right)$ [2, Theorem 2.6] for $1<p<\infty$.

We now consider a consequence of Theorem 3.2. First we need to introduce some more notation. Given a sequence $\left\{R_{N}(\lambda): N \in N\right\}$ 
of functions of $\lambda \in R^{*}$ define the following difference-differential operators:

$$
\begin{aligned}
& D_{+} R_{N}(\lambda)=[(N+1) / 2|\lambda|]^{1 / 2}\left[R_{N+1}(\lambda)-R_{N}(\lambda)\right] \\
& D_{-} R_{N}(\lambda)=[N / 2|\lambda|]^{1 / 2}\left[R_{N}(\lambda)-R_{N-1}(\lambda)\right] \\
& D_{1} R_{N}(\lambda)=-R_{N}^{\prime}(\lambda)+(N / \lambda)\left[R_{N}(\lambda)-R_{N-1}(\lambda)\right] \\
& D_{2} R_{N}(\lambda)=-R_{N}^{\prime}(\lambda)+[(N+1) / \lambda]\left[R_{N+1}(\lambda)-R_{N}(\lambda)\right],
\end{aligned}
$$

where $R_{N}^{\prime}(\lambda)$ denotes the derivative of $R_{N}(\lambda)$ with respect to $\lambda$. We think of $D_{+}$and $D_{-}$as "operators of order one" and $D_{1}$ and $D_{2}$ as "operators of order two". For every positive integer $j$ let $\Delta^{j}$ denote any product of order $j$ of the operators $D_{+}, D_{-}, D_{1}$ and $D_{2}$. So, for instance, $\Delta^{1}$ denotes either $D_{+}$or $D_{-}, \Delta^{2}$ denotes either $D_{1}$ or $D_{2}$ or $D_{+} D_{-}$and so on. Let $\mathscr{D}^{n}$ be the product $\mathscr{D}_{2} \cdots \mathscr{D}_{n}$. Finally let $\Pi_{s}^{2}, s>0$, be the projection $\sum P_{N}^{(n)}(\lambda)(s \leqq(2 N+1)|\lambda|<2 s)$.

Then iterating Theorem 3.2 and specializing the multiplier theorem in [4] to the case of zonal multipliers, we get:

CoRollary 3.4. Let $\left\{R_{N}(\lambda): N \in N\right\}$ be a sequence of uniformly bounded functions in $C^{2}\left(\boldsymbol{R}^{*}\right)$ such that

$$
\left.\sup _{s>0} \int_{-\infty}^{+\infty}|| \prod_{s}^{2} \Delta^{j} \mathscr{D}^{n-1} R_{N}(\lambda)\right|_{H S} ^{2}|\lambda| d \lambda \leqq \mathscr{C} \quad s^{2-j}
$$

for every $j=0, \cdots, 4$. Then the zonal multiplier defined by the operator valued function $M(\lambda)=\sum_{N \in N} R_{N}(\lambda) P_{N}^{(n)}(\lambda)$ is bounded on $L^{p}\left(\boldsymbol{H}^{n}\right), 1<p<\infty$.

4. An algebra of zonal multipliers. Let $\mathscr{S}^{n}$ be the Lie algebra of $\boldsymbol{H}^{n}$. A basis of $\mathfrak{S}^{n}$ is given by the left invariant vector fields:

$$
T=\frac{\partial}{\partial t}, Z_{j}=\frac{\partial}{\partial z_{j}}+i \bar{z}_{j} \frac{\partial}{\partial t}, \bar{Z}_{j}=\frac{\partial}{\partial \bar{z}_{j}}-i z_{j} \frac{\partial}{\partial t} \quad j=1, \cdots, n .
$$

The differential operator $T$ spans the center of $\mathfrak{S}^{n}$ and its Fourier transform is $d \pi_{\lambda}(T)=i \lambda I$, where $d \pi_{\lambda}$ denotes the derived representation of $\pi_{\lambda}$. It is also clear that $T$ is invariant under the action of $S U(n)$. Next consider the left invariant differential operator

$$
\mathscr{L}=\frac{1}{2} \sum_{j}\left(Z_{j} \bar{Z}_{j}+\bar{Z}_{j} Z_{j}\right) .
$$

It is well known that $\mathscr{L}$ is hypoelliptic and that it plays much the same fundamental role on $\boldsymbol{H}^{n}$ as the Laplacian does on $\boldsymbol{R}^{n}$ [6]. In particular $\mathscr{L}$ is $S U(n)$ invariant. Its Fourier transform is [7]:

$$
d \pi_{\lambda}(\mathscr{L})=\sum_{N \in N}(2 N+n)|\lambda| P_{N}^{(n)}(\lambda) \quad \lambda \in \boldsymbol{R}^{*} .
$$


Denote now by $S_{n}$ the sector $\left\{(\lambda, r) \in \boldsymbol{R}^{2}: r \geqq n|\lambda|>0\right\}$. Let $\mathscr{F}_{n}$ be the family of all functions $F$ in $C^{n+3}\left(S_{n}\right)$ such that

$$
\left|\partial^{l} F(x)\right| \leqq C|x|^{-|l|}, \quad x \in S_{n}
$$

for all pairs $l \in N^{2}$ such that $|l|=l_{1}+l_{2} \leqq n+3$. Given a function $F \in \mathscr{F}_{n}$ let $M_{F}(-i T, \mathscr{L})$ be the multiplier defined by

$$
M_{F}(-i T, \mathscr{L})(\lambda)=\sum_{N \in N} F(\lambda,(2 N+n)|\lambda|) P_{N}^{(n)}(\lambda) \quad \lambda \in R^{*} .
$$

Then $\mathscr{C}_{n}=\left\{M_{F}(-i T, \mathscr{L}): F \in \mathscr{F}_{n}\right\}$ is a *algebra of zonal multipliers of $L^{2}\left(\boldsymbol{H}^{n}\right)$.

THEOREM 4.1. The set $\mathscr{L}_{n}$ is a ${ }^{*}$-algebra of zonal multipliers of $L^{p}\left(\boldsymbol{H}^{n}\right), 1<p<\infty$.

Proof. The proof is by induction on $n$. The case $n=1$ follows from Corollary 3.4 using Taylor's formula to express the differencedifferential operators in (3.3) in terms of derivatives of $F$. We consider in detail only the case of $D_{1}$ which is already entirely typical. Let $r=(2 N+1)|\lambda|$. Then:

$$
\begin{aligned}
D_{1} F(\lambda, r)=- & \partial_{\lambda} F(\lambda, r)-(2 N+1) \operatorname{sign}(\lambda) \partial_{r} F(\lambda, r) \\
& +\frac{N}{\lambda}[F(\lambda, r)-F(\lambda, r-2|\lambda|)]
\end{aligned}
$$

for $N \geqq 1$ and

$$
D_{1} F(\lambda, r)=-\partial_{\lambda} F(\lambda, r)-\operatorname{sign}(\lambda) \partial_{r} F(\lambda, r)
$$

for $N=0$. Hence for $N \geqq 1$ :

$$
\begin{gathered}
D_{1} F(\lambda, r)=-\partial_{\lambda} F(\lambda, r)-(2 N+1) \operatorname{sign}(\lambda) \partial_{r} F(\lambda, r) \\
+\frac{N}{\lambda} \int_{r-2|\lambda|}^{r}\left(\partial_{r} F(\lambda, r)+\int_{r}^{s} \partial_{r}^{2} F(\lambda, t) d t\right) d s .
\end{gathered}
$$

Since $r \geqq|\lambda|$ and, for $N \geqq 1, r-2|\lambda| \geqq r / 3$, from (4.1) follows that

$$
\left|D_{1} F(\lambda,(2 N+1)|\lambda|)\right| \leqq C^{\prime}(2 N+1)^{-1}|\lambda|^{-1} \text { for } N \in N \text {. }
$$

It is not hard to see now that (4.2) implies (3.3) for $4^{2}=D_{1}$.

Next assume the theorem proved for $n-1, n \geqq 2$. By Theorem 3.2 the operator $M(-i T, \mathscr{L})$ is bounded on $L^{p}\left(\boldsymbol{H}^{n}\right), 1<p<\infty$, provided that the multiplier

$$
m(\lambda)=\sum_{N \in N} \mathscr{D}_{n} F(\lambda,(2 N+n)|\lambda|) P_{N}^{(n-1)}(\lambda) \quad \lambda \in \boldsymbol{R}^{*}
$$

is bounded on $L^{p}\left(\boldsymbol{H}^{n-1}\right)$. We claim that $m$ is of the form $M_{G}(-i T, \mathscr{L})$ 
where $G \in \mathscr{F}_{n-1}$. Indeed let $G$ be the function defined by $G(\lambda, r)=\frac{r}{2} \int_{-1}^{1} \partial_{r} F(\lambda, r+t|\lambda|)+\frac{n-1}{2}[F(\lambda, r+|\lambda|)-F(\lambda, r-|\lambda|)]$ for $r \geqq(n+1)|\lambda|$, and by

$$
G(\lambda, r)=(n-1) F(\lambda, r+|\lambda|)
$$

for $(n-1)|\lambda| \leqq r \leqq n|\lambda|$ and still undefined for $n|\lambda|<r<(n+1)|\lambda|$. It is easily seen that: $\mathscr{D}_{n} F(\lambda,(2 N+n)|\lambda|)=G(\lambda,(2 N+n-1)|\lambda|)$ for $\lambda \in \boldsymbol{R}^{*}, N \in N$. Moreover $G$ is $n+2$ times differentiable and $\left|\partial^{l} G(x)\right| \leqq C|x|^{-|l|}$ for $|l| \leqq n+2$ for every $x=(\lambda, r)$ such that either $0<(n-1)|\lambda| \leqq r \leqq n|\lambda|$ or $0<(n+1)|\lambda| \leqq r$. Therefore, in order to complete the proof of the theorem, we need only to show that the function $G$ can be extended to a $C^{n+2}$ function satisfying the inequalities (4.1) in all the sector $S_{n-1}$. This is precisely the purpose of the following lemma.

Lemma 4.2. Let $\mathscr{F}$ be a region in $\boldsymbol{R}^{2}$ which is the union of two cones $\mathscr{F}_{1}=\left\{(\lambda, r): c_{1} r \leqq \lambda \leqq c_{2} r, r, \lambda>0\right\}$ and $\mathscr{F}_{2}=\left\{(\lambda, r): c_{3} r \leqq\right.$ $\left.\lambda \leqq c_{4} r, r, \lambda>0\right\}$ where $0 \leqq c_{1}<c_{2}<c_{3}<c_{4}$. Assume that $f$ is a function in $C^{k}(\mathscr{F})$ such that

$$
\left|\partial^{l} f(x)\right| \leqq c|x|^{-|l|} \quad x \in \mathscr{F}
$$

whenever $|l|=l_{1}+l_{2} \leqq k$. Then there exists a function $E(f)$ defined and $k$ times continuously differentiable in the region $\mathscr{G}=\{(\lambda, r)$ : $\left.c_{1} r \leqq \lambda \leqq c_{4} r, r, \lambda>0\right\}$ which extends $f$ and satisfies

$$
\left|\partial^{l} E f(x)\right| \leqq C|x|^{-|l|} \quad x \in \mathscr{G}
$$

whenever $|l| \leqq k$.

Proof. The proof is based on Whitney's extension theorem. Let $\Omega$ be the region between the two cones, i.e., $\Omega=\left\{(\lambda, r): 0<c_{z} r<\right.$ $\left.\lambda<c_{3} r\right\}$. Then there exists a collection of squares with sides parallel to the axes $Q_{1}, Q_{2}, \cdots, Q_{k}, \cdots$ such that

(1) $U_{k} Q_{k}=\Omega$.

(2) the $Q_{k}$ are mutually disjoint.

(3) $\operatorname{diam}\left(Q_{k}\right) \leqq \operatorname{dist}\left(Q_{k}, \mathscr{F}\right) \leqq 4 \operatorname{diam}\left(Q_{k}\right)$.

For the proof of this and the following facts we refer the reader to Chapter VI of Stein's book [13]. Denote by $Q_{k}^{*}$ the square which has the same center as $Q_{k}$, but is expanded by a factor $(1+\varepsilon)$, where $\varepsilon$ is a fixed number, $0<\varepsilon<1 / 4$. Then each point of $\Omega$ is contained in at most a finite number $N$ of the cubes $Q_{k}^{*}$. Given the covering $\left\{Q_{k}^{*}\right\}$ of $\Omega$ there exists a partition of the identity $\left\{\varphi_{l}^{*}\right\}$ subordinated 
to it such that

$$
\left|\partial^{\alpha} \varphi_{k}^{*}(x)\right| \leqq A\left(\operatorname{diam} Q_{k}\right)^{-|\alpha|} .
$$

For each square $Q_{k}$ fix a point $p_{k} \in \mathscr{F}$ of minimum distance of $\mathscr{F}$ from $Q_{k}$. Notice that such point exists, even if $\mathscr{F}$ is not closed because the origin is not in $\mathscr{F}$. The definition of $E(f)$ is as follows:

$$
\begin{aligned}
& E(f)(x)=f(x) \quad \text { if } \quad x \in \mathscr{F} \\
& E(f)(x)=\sum_{i} P\left(x, p_{i}\right) \varphi_{i}^{*}(x) \quad x \in \Omega
\end{aligned}
$$

where $P\left(x, p_{i}\right)$ is the polynomial giving the Taylor expansion of $f$ about the point $p_{i}$, that is

$$
P\left(x, p_{i}\right)=\sum_{|l| \leqq k} \frac{\partial^{l} f\left(p_{i}\right)}{l !}\left(x-p_{\imath}\right)^{l} \quad x \in \Omega .
$$

It is then clear by Whitney's theorem [13, Ch, VI, 4.7], that $E(f) \epsilon$ $C^{k}(\mathscr{F} \cup \Omega)$. It remains only to show that the inequalities (4.3) are satisfied. Remark that by (4.2)

$$
\partial^{j} f(x)=\sum_{|l+j| \leqq k-1} \frac{\partial^{j+l} f(y)}{l !}(x-y)^{l}+R_{j}(x, y) \quad|j| \leqq k-1
$$

and

$$
\partial^{j} f(x)=\partial^{j} f(y)+R_{j}(x, y) \quad|j|=k
$$

where $x, y \in \mathscr{F}$ and $\mid R_{j}\left(x, y\left|\leqq c \max \left\{|x|^{-k},|y|^{-k}\right\}\right| x-\left.y\right|^{k-|j|}\right.$. Now let $P_{j}(x, y)$ be the Taylor polynomial of $\partial^{j} f$ about the point $y$, i.e.:

$$
P_{j}(x, y)=\sum_{|l+j| \leqq k} \frac{\partial^{j+l} f(y)}{l !}(x-y)^{l} \quad y \in \mathscr{F}, \quad x \in \boldsymbol{R}^{2} .
$$

Then as in [13, p. 177] it is not hard to see that

$$
P_{j}(x, b)-P_{j}(x, a)=\sum_{|j+l| \leqq k} R_{j+l}(b, a)-\frac{(x-b)^{l}}{l !}
$$

for $a, b \in \mathscr{F}, x \in \Omega$. Now since $\operatorname{dist}\left(Q_{2}^{*}, \mathscr{F}\right)$ is comparable with $\operatorname{diam}\left(Q_{i}\right)$ there exist positive constants $d_{1}, d_{2}, d_{3}$ so that for $x \in$ $Q_{2}^{*}\left|x-p_{i}\right| \leqq d_{1}\left|p_{i}\right|$ and $d_{2}\left|p_{i}\right| \leqq|x| \leqq d_{3}\left|p_{i}\right|$.

Let $|j| \leqq k$ and consider $\partial^{j} E(f)(x)$ for $x \in \Omega$. By (4.4):

$$
\partial^{j} E(f)(x)=\sum_{i} \partial^{j} P\left(x, p_{i}\right) \varphi_{i}^{*}(x)+\text { other terms } .
$$

Disregrading the other terms for the moment and observing that $\partial^{j} P\left(x, p_{i}\right)=P_{j}\left(x, p_{i}\right)$ we get: 


$$
\left|\sum_{i} \partial^{j} P\left(x, p_{i}\right) \Phi_{i}^{*}(x)\right| \leqq \sum_{i} \sum_{|l+j| \leqq k} \frac{\partial^{j+l} f\left(p_{i}\right)}{l !}\left|x-p_{i}\right|^{|l|} \leqq c|x|^{-|j|} .
$$

The other terms are themselves sums of expressions like:

$$
\sum_{i} P_{j-l}\left(x, p_{i}\right) \partial^{l} \varphi_{i}^{*}(x)
$$

where $0<|l|, l \leqq j$. Since $\sum_{i} \partial^{l} \varphi_{i}^{*}(x)=0$ for $x \in \Omega$ these sums are in turn equal to:

$$
\sum_{\imath}\left[P_{j-l}\left(x, p_{i}\right)-P_{j-l}(x, a)\right] \partial^{j} \varphi_{i}^{*}(x) \quad a \in \mathscr{F} .
$$

Choose $a$ to be the point of minimum distance of $x$ from $\mathscr{F}$. Then since the angle between $\mathscr{F}_{1}$ and $\mathscr{F}_{2}$ is less than $\pi / 2$, there exists a constant $C_{0}>0$ such that $C_{0}|x| \leqq|a| \leqq|x|$. Notice also that we have $\left|a-p_{i}\right| \leqq 2\left|x-p_{i}\right|$. Therefore by (4.4), (4.6) the expression (4.7) can be majorized by:

$$
c^{\prime} \sum_{i} \max \left(|a|^{-k},\left|p_{i}\right|^{-k}\right)\left|x-p_{i}\right|^{k-|j|}
$$

which in turn is not larger than $c^{\prime \prime}|x|^{-|j|}$.

5. Multipliers and group contractions. In this section we study a relationship between $L^{p}$ multipliers on $\boldsymbol{H}^{n}$ and $L^{p}$ multipliers on $\boldsymbol{C}^{n}$, with the aim of obtaining necessary conditions for the boundedness of a multiplier on $L^{p}\left(\boldsymbol{H}^{n}\right)$. The main tool here is the contraction of $\boldsymbol{H}^{n}$ to $\boldsymbol{C}^{n}$ introduced by Geller in [8].

Let

$$
f(t, z)=\sum_{m} f_{m}(t, r) \exp (i\langle m, \theta\rangle)
$$

where $r \in \boldsymbol{R}_{+}^{n}, \theta \in[0,2 \pi]^{n}, m \in \boldsymbol{Z}^{n}$ and $z_{k}=r_{k} e^{i \theta_{k}}$, be a function in $\mathscr{S}\left(\boldsymbol{H}^{n}\right)$ and consider its Fourier transform $\hat{f}(\lambda)=\sum_{m, \alpha} R_{\alpha}^{m}(\lambda ; f) W_{\alpha}^{m}(\lambda)$. The contraction process on the Fourier transform side consists in taking the limit of $R_{\alpha}^{m}(\lambda ; f)$ as $\lambda \rightarrow 0$ and $\alpha \rightarrow+\infty$ in such a way that $\left(2 \alpha_{j}+n\right)|\lambda| \rightarrow r_{j}^{2}$, for $j=1, \cdots, n$. Let $R^{m}\left(r_{1}, \cdots, r_{n} ; f\right)$ be this limit. It is well known that for $k>0$ the Bessel function of order $k J_{k}(2 r \rho)$ is the limit as $\lambda \rightarrow 0$ and $2 j|\lambda| \rightarrow \rho$ of $l_{j}^{k}\left(2|\lambda| r^{2}\right)$, uniformly for $\rho$ in a compact set. Therefore from (2.2) and the Bessel transform formulas [14, Ch. 2, Theorem 1.6] the function

$$
\sum_{m \in Z^{n}}(-1)^{\mid m+} R^{m}\left(r_{1}, \cdots, r_{n} ; f\right) \exp (i\langle m, \theta-\pi / 2\rangle)
$$

is the Euclidean Fourier transform of the contracted function $f^{b}(z)=$ $\int_{-\infty}^{+\infty} f(t, z) d t$. 
The contraction process has a right inverse. Given a function $\varphi \in \mathscr{S}\left(\boldsymbol{C}^{n}\right)$ such that

$$
\varphi(\zeta)=\sum_{m}(-1)^{|m+|} \varphi_{m}\left(\rho_{1}^{2} / 4, \cdots, \rho_{n}^{2} / 4\right) \exp (i\langle m, \theta-\pi / 2\rangle)
$$

where $\zeta_{k}=\rho_{k} e^{i \theta_{k}},\langle m, \theta\rangle=\sum_{k} m_{k} \theta_{k}$, the operator valued function

$$
\varphi^{*}(\lambda)=\sum_{\alpha, m} \varphi_{m}\left(\left(2 \alpha_{1}+n\right)|\lambda|, \cdots,\left(2 \alpha_{n}+n\right)|\lambda|\right) W_{\alpha}^{m}(\lambda)
$$

is the (noncommutative) Fourier transform of a function $f \in \mathscr{S}\left(\boldsymbol{H}^{n}\right)$, which contracts to the inverse (Euclidean) Fourier transform of $\varphi$.

Now let $M$ be a bounded function on $C^{n}$ such that

$$
M(\zeta)=\sum_{m \in \mathbf{Z}^{n}}(-1)^{\left|m^{+}\right|} M_{m}\left(r_{1}^{2} / 4, \cdots, r_{n}^{2} / 4\right) \exp \left(i\left\langle m, \theta-\frac{\pi}{2}\right\rangle\right), \quad \zeta \in C^{n},
$$

where as before, $\zeta_{j}=r_{j} e^{i \theta_{j}}$ and $M_{m}$ are for every $m \in Z^{n}$ almost everywhere continuous on $\boldsymbol{R}_{+}^{n}$. For every $\varepsilon>0$ define the operator valued function $M_{\varepsilon}$ by:

$$
M_{\varepsilon}(\lambda)=\sum_{m, \alpha} M_{m}\left(\left(2 \alpha_{1}+n\right)|\lambda| \varepsilon, \cdots,\left(2 \alpha_{n}+n\right)|\lambda| \varepsilon\right) W_{\alpha}^{m}(\lambda) \quad \lambda \in \boldsymbol{R}^{*} .
$$

TheOREM 5.1. If $M_{\varepsilon}$ is for every $\varepsilon>0$ a multiplier or $L^{p}\left(\boldsymbol{H}^{n}\right)$ with norm independent of $\varepsilon$, then $M$ is a multiplier of $L^{p}\left(\boldsymbol{C}^{n}\right)$. Moreover $\|M\|_{\mathrm{Convp}_{\boldsymbol{p}}\left(\boldsymbol{C}^{n}\right)} \leqq 2 \pi \lim \sup _{\varepsilon \rightarrow 0}\left\|M_{\varepsilon}\right\|_{\mathrm{Conv}\left(\boldsymbol{H}^{n}\right)}$.

Proof. Let $q$ be the index conjugate to $p$. It suffices to show that $I=\int_{\boldsymbol{c}^{n}} M f(z) g(z) d z d \bar{z}$ is bounded in absolute value by $c\|f\|_{p}\|g\|_{q}$ whenever $f, g \in C_{c}\left(C^{n}\right)$, with $c$ independent of $f$ and $g$. We may assume that $M_{m}$ decreases rapidly, say

$$
\left|M_{m}\left(r_{1}^{2}, \cdots, r_{n}^{2}\right)\right| \leqq c_{1} \exp \left[-c_{2}\left(|m|^{2}+r^{2}\right)\right],
$$

where $r^{2}=\sum_{i} r_{i}^{2}$, replacing if necessary the multiplier $M$ by the multiplier $M^{\sigma}(\sigma>0)$ defined by

$$
\begin{aligned}
M^{\sigma}(\zeta)= & \sum_{m}(-1)^{|m+|} M_{m}\left(r_{1}^{2} / 4, \cdots, r_{n}^{2} / 4\right) \exp \left[-\sigma\left(|m|^{2}+r^{2}\right)\right. \\
& +i\langle m, \theta-\pi / 2\rangle]
\end{aligned}
$$

and observing that the constant $c$ will not depend on $\sigma$. Therefore we may apply Fubini's theorem and the Fourier-Bessel transform to write:

$$
\begin{aligned}
I= & (2 \pi)^{-n} \sum_{m}(-1)^{\left|m^{-}\right|} \exp \left(i\left\langle m, \theta^{\prime}\right\rangle\right) \int_{\boldsymbol{C}^{n}} \int_{C^{n}} \int_{\boldsymbol{R}_{+}^{n}} M_{m}\left(\rho_{1}^{2} / 4, \cdots, \rho_{n}^{2} / 4\right) \\
& \times \prod_{k} J_{m_{k}}\left(r_{k}^{\prime} \rho_{k}\right) \rho_{k} d \rho_{k} f\left(z-z^{\prime}\right) g(z) d z^{\prime} d \bar{z}^{\prime} d z d \bar{z}
\end{aligned}
$$


with obvious notation. Now choose two functions $\varphi$ and $\psi$ in the Schwartz space $\mathscr{S}(\boldsymbol{R})$ such that $\|\varphi\|_{p}=\left\|\psi_{\psi}\right\|_{q}=1$ and $\int \hat{\varphi}(\lambda) \hat{\psi}(-\lambda) d \lambda=$ 1 , and define $f_{\varepsilon}^{\sharp}(t, z)=\varepsilon^{-n / 2} \varphi(t) f\left(\varepsilon^{-1 / 2} z\right) ; g_{\varepsilon}^{\sharp}(t, z)=\varepsilon^{-n / 2} \psi(t) g\left(\varepsilon^{-1 / 2} z\right)$ for $(t, z) \in \boldsymbol{H}^{n}$. Then $f_{\varepsilon}^{\sharp} \in L^{p}\left(\boldsymbol{H}^{n}\right), g_{\varepsilon}^{\sharp} \in L^{q}\left(\boldsymbol{H}^{n}\right)$ and $\left\|f_{\varepsilon}^{\sharp}\right\|_{p}=\varepsilon^{n(1 / p-1 / 2)}\|f\|_{p}$, $\left\|g_{\varepsilon}^{*}\right\|_{q}=\varepsilon^{n(1 / q-1 / 2)}\|g\|_{q}$.

Let $I_{\varepsilon}=\int_{\boldsymbol{H}^{n}} M_{\varepsilon} f_{\varepsilon}^{\sharp} g_{\varepsilon}^{\sharp} d t d z d^{\bar{z}}$. By hypothesis there exists $A$, independent of $\varepsilon, f, g$, such that $\left|I_{\varepsilon}\right| \leqq A\left\|f_{\varepsilon}^{\sharp}\right\|_{p}\left\|g_{\varepsilon}^{\sharp}\right\|_{q}$. We claim that there exists a constant $c$ depending only on $n$ such that $\lim _{\varepsilon \rightarrow 0} I_{\varepsilon}=c I$. In fact:

$$
\begin{aligned}
I_{\varepsilon}= & \varepsilon^{-n} \frac{2^{n-1}}{\pi^{n+1}} \int_{H^{n}} \int_{t^{n} n} \int_{-\infty}^{+\infty} \sum_{m} e^{-i \lambda t^{\prime}} \sum_{m, \alpha} e^{i\left\langle m, \theta^{\prime}\right\rangle} M_{m}\left(\left(2 \alpha_{k}+n\right)|\lambda| \varepsilon\right) \\
& \times \prod_{k} l_{\alpha_{k}}^{\left|m_{k}\right|}\left(2|\lambda|\left|z_{k}^{\prime}\right|^{2}\right) \rho\left(t-t^{\prime}-\Phi\left(z, z^{\prime}\right)\right) \psi(t) f\left(\varepsilon^{-1 / 2}\left(z-z^{\prime}\right)\right) g\left(\varepsilon^{-1 / 2} z\right) \\
& \times|\lambda|^{n} d \lambda d t d z d \bar{z} d t^{\prime} d z^{\prime} d \bar{z}^{\prime} .
\end{aligned}
$$

Making the change of variables $\zeta=\varepsilon^{-1 / 2} z, \zeta^{\prime}=\varepsilon^{-1 / 2} z^{\prime}$ and performing the integrations in $t$ and $t^{\prime}$, we get:

$$
\begin{aligned}
I_{\varepsilon}= & \varepsilon^{n} \frac{2^{n-1}}{\pi^{n+1}} \int_{C^{n}} \int_{C^{n}} \int_{-\infty}^{+\infty} e^{i\left\langle m, \theta^{\prime}\right\rangle} e^{i \lambda \varepsilon n \Phi\left(\zeta, \zeta^{\prime}\right)} \widehat{\varphi}(\lambda) \hat{\psi}(-\lambda) \\
& \times \sum_{\alpha} M_{m}\left(\left(2 \alpha_{k}+n\right)|\lambda| \varepsilon\right) \prod_{k} l_{\alpha_{k}}^{\left|m_{k}\right|}\left(2|\lambda| \varepsilon\left|\zeta_{k}^{\prime}\right|^{2}\right) \\
& \times f\left(\zeta-\zeta^{\prime}\right) g(\zeta)|\lambda|^{n} d \lambda d \zeta d \bar{\zeta} d \zeta^{\prime} d \bar{\zeta}^{\prime} .
\end{aligned}
$$

Now consider the sum

$$
\varepsilon^{n}|\lambda|^{n} \sum_{\alpha} M_{m}\left(\left(2 \alpha_{1}+n\right)|\lambda| \varepsilon, \cdots,\left(2 \alpha_{n}+n\right)|\lambda| \varepsilon\right) \prod_{k} l_{\alpha_{k}}^{m_{k}}\left(2|\lambda| \varepsilon\left|\zeta_{k}^{\prime}\right|^{2}\right) .
$$

From the asymptotic formula of Hilb's type [15, Theorem 8.22.4] it follows that $\lim l_{\alpha_{k}}^{m_{k} \mid}\left(2|\lambda| \varepsilon\left|\zeta_{k}^{\prime}\right|^{2}\right)=(-1)^{m_{k}^{-}} J_{m_{k}}\left(2\left|\zeta_{k}^{\prime}\right| \rho_{k}\right)$ as $\varepsilon \rightarrow 0, \alpha_{k} \rightarrow \infty$ in such a way that $\left(2 \alpha_{k}+n\right)|\lambda| \varepsilon \rightarrow \rho_{k}$ uniformly for $\zeta_{k}$ in a compact set. Therefore (5.1) is a Riemann sum approximation to:

$$
2^{-n}(-1)^{m_{k}^{-}} \int_{R^{n}} M_{m}\left(\rho_{1}^{2} / 4, \cdots, \rho_{n}^{2} / 4\right) \prod_{k} J_{m_{k}}\left(\rho_{k}\left|\zeta_{k}^{\prime}\right|\right) \rho_{k} d \rho_{k} .
$$

Since the functions $M_{m}$ are almost everywhere continuous and fast decreasing both in the $m$ and $\rho$ variables, (5.1) tends to (5.2) as $\varepsilon \rightarrow 0$. Therefore $\lim _{\varepsilon \rightarrow 0} I_{\varepsilon}=(2 \pi)^{-1} I$ as claimed.

This proves the theorem.

\section{REFERENCES}

1. R. Coifman and G. Weiss, Analyse harmonique non commutative sur certaines éspaces homogènes, Lecture Notes in Math., vol. 242, Springer-Verlag, Berlin, 1971. 
2. R. Coifman and G. Weiss, Transference methods in analysis, CBMS Regional Conference Series in Mathematics, No. 31, Amer. Math. Soc., (1977).

3. L. De Michele and G. Mauceri, $L^{p}$ multipliers on the Heisenberg group, Proc. Symposia Pure Math. Amer. Math. Soc., vol. 35 (1979).

4. ——, $L^{p}$ multipliers on the Heisenberg group, to appear in Michigan Math. J.

5. A. Erdelyi et Al., Higher Transcendental Functions, Vol. II, Bateman Manuscript Project, McGraws-Hill, New York, 1955.

6. G. B. Folland, Subelliptic estimates and function spaces on nilpotent Lie groups, Ark. Math., 13 (1975), 161-207.

7. G. B. Folland and M. E. Stein, Estimates for the $\vec{\partial}_{b}$ complex and analysis on the Heisenberg group, Comm. Pure Appl. Math., 27 (1974), 429-522.

8. D. Geller, Fourier analysis on the Heisenberg group, Ph. D. Thesis, Princeton University, (1977).

9. D. Geller, Fourier analysis on the Heiseberg group, Proc. Natl. Acad. Sci. USA, 74 No. 4, (1977), 1328-1331.

10. C. Herz, The theory of p-spaces with an application to convolution operators, Trans. Amer. Math. Soc., 154 (1971), 69-82.

11. C. Herz, Une generalisation de la notion de transformée de Fourier-Stieltjes, Ann. Inst. Fourier, Grenoble, 24, 3 (1974), 145-157.

12. A. Koranyi, S. Vagi and G. V. Welland, Remarks on the Cauchy integral and the conjugate function in generalized half-planes, J. Math. and Mech., 19, no. 12, (1970), 1069-1081.

13. E. M. Stein, Singular Integrals and Differentiability Properties of Functions, Princeton Univ. Press, Princeton, N. J., 1970.

14. E. M. Stein and G. Weiss, Introduction to Fourier Analysis on Euclidean Spaces, Princeton Univ. Press, Princeton, N. J., 1971.

15. G. Szego, Orihogonal polynomials, Amer. Math. Soc. Colloq. Publ., Vol. 23, Amer. Math. Soc., Providence, R. I., 1939.

Received July 31, 1979 and in revised form June 24, 1980. This paper was written while the author was visiting the University of California, Berkeley with a fellowship from Consiglio Nazionale delle Ricarche.

UNIVERSITA DI GENOVA

16132 Genova, Italy 



\section{PACIFIC JOURNAL OF MATHEMATICS}

\section{EDITORS}

DONALD BABBITT (Managing Editor)

University of Galifornia

Los Angeles, California 90024

Hugo RossI

University of Utah

Salt Lake City, UT 84112

C. C. MOORE AND ANDREW OGG

University of California

Berkeley, CA 94720
J. DugundJI

Department of Mathematics University of Southern California Los Angeles, California 90007

R. FinN and J. Milgram Stanford University Stanford, California 94305

\section{ASSOCIATE EDITORS}

R. ARENS

E. F. BeCKenbaCh

B. H. NEUManN

F. WOLF

K. YosHIDA

\section{SUPPORTING INSTITUTIONS}

UNIVERSITY OF ARIZONA

UNIVERSITY OF BRITISH COLUMBIA

CALIFORNIA INSTITUTE OF TECHNOLOGY

UNIVERSITY OF CALIFORNIA

MONTANA STATE UNIVERSITY

UNIVERSITY OF NEVADA, RENO

NEW MEXICO STATE UNIVERSITY

OREGON STATE UNIVERSITY
UNIVERSITY OF OREGON

UNIVERSITY OF SOUTHERN CALIFONIA

STANFORD UNIVERSITY

UNIVERSITY OF HAWAII

UNIVERSITY OF TOKYO

UNIVERSITY OF UTAH

WASHINGTON STATE UNIVERSITY

UNIVERSITY OF WASHINGTON 


\section{Pacific Journal of Mathematics}

\section{Vol. 95, No. $1 \quad$ September, 1981}

John Allen Beachy and William David Blair, On rings with bounded

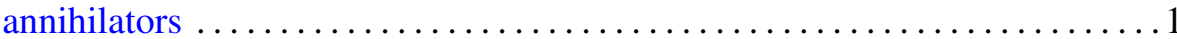

Douglas S. Bridges, A constructive look at positive linear functionals on

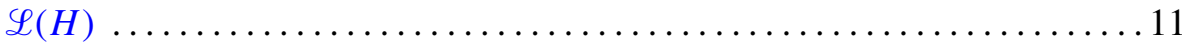

Muneo Chō and Makoto Takaguchi, Boundary points of joint numerical ranges

W. J. Cramer and William O. Ray, Solvability of nonlinear operator equations

Lester Eli Dubins and Gideon Schwarz, Equidiscontinuity of

Borsuk-Ulam functions

Maria Fragoulopoulou, Spaces of representations and enveloping 1.m.c.

*-algebras

Robert F. Geitz and J. Jerry Uhl, Jr., Vector-valued functions as families of scalar-valued functions

Ross Geoghegan, The homomorphism on fundamental group induced by a homotopy idempotent having essential fixed points

Ross Geoghegan, Splitting homotopy idempotents which have essential fixed points

Paul Jacob Koosis, Entire functions of exponential type as multipliers for

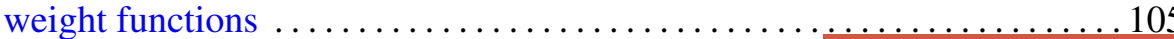

David London, Monotonicity of permanents of certain doubly stochastic matrices

Howard J. Marcum, Two results on cofibers

Giancarlo Mauceri, Zonal multipliers on the Heisenberg group

Edward Wilfred Odell, Jr. and Y. Sternfeld, A fixed point theorem in $c_{0} \quad \ldots 161$

Bernt Karsten Oksendal, Brownian motion and sets of harmonic measure zero

Andrew Douglas Pollington, The Hausdorff dimension of a set of normal numbers

Joe Repka, Base change lifting and Galois invariance ...

Gerald Suchan, Concerning the minimum of permanents on doubly stochastic circulants

Jun-ichi Tanaka, On isometries of Hardy spaces on compact abelian groups

Aaron R. Todd, Quasiregular, pseudocomplete, and Baire spaces 